\title{
Combined Electrical and Optical Analysis of the Efficiency Roll-Off in Phosphorescent Organic Light-Emitting Diodes
}

\author{
Sebastian Wehrmeister, ${ }^{1,}$ Lars Jäger, ${ }^{1}$ Thomas Wehlus, ${ }^{2}$ Andreas F. Rausch, ${ }^{2}$ Thilo C. G. Reusch, ${ }^{2}$ \\ Tobias D. Schmidt, ${ }^{1}$ and Wolfgang Brütting ${ }^{1, \dagger}$ \\ ${ }^{1}$ Institute of Physics, University of Augsburg, 86135 Augsburg, Germany \\ ${ }^{2}$ OSRAM OLED GmbH, 93049 Regensburg, Germany \\ (Received 5 September 2014; revised manuscript received 3 December 2014; published 19 February 2015)
}

In this paper, we present a method for a comprehensive analysis of the efficiency roll-off with current density in phosphorescent organic light-emitting diodes (OLEDs). By combining electrical and optical excitation in time-resolved spectroscopic experiments, we are able to measure the excited-state lifetime for different driving conditions. It is, thus, possible to correlate changes of the triplet lifetime with a decrease of the radiative quantum efficiency of the emitting system due to exciton quenching processes. As compared to the conventional analysis of the measured external quantum efficiency (EQE) in dependence of the applied current density, the lifetime analysis is not affected by changes of the charge-carrier balance with current, which can have a significant impact on the interpretation of the results. By performing timeresolved spectroscopy for a series of red phosphorescent OLEDs, triplet-polaron quenching (TPQ) is identified as the dominant mechanism behind the efficiency roll-off up to a current density of $100 \mathrm{~mA} / \mathrm{cm}^{2}$, while the conventional EQE vs current plot rather suggests triplet-triplet annihilation as the main quenching mechanism. We show that this apparent discrepancy is caused by exciton quenching occurring already at very low current densities, where $\mathrm{EQE}$ measurements are not reliable due to significant changes of the charge-carrier balance in this region. In addition, we present evidence that the triplet-polaron quenching rate $\Gamma_{\mathrm{TPQ}}$ is independent of the microcavity so that variations of the triplet lifetimes of a series of devices exhibiting different layer thicknesses can be described with a single parameter set.

DOI: 10.1103/PhysRevApplied.3.024008

\section{INTRODUCTION}

Starting with the first thin-film organic light-emitting diode (OLED) presented by Tang and VanSlyke [1] in 1987, the research on OLEDs has made tremendous progress. The use of multilayer structures with conductivitydoped transport layers and selective carrier-blocking layers as well as the introduction of highly efficient electrophosphorescent guest-host systems mark only a few of these improvements resulting in higher efficiencies [2-5]. The increasing number of commercial applications based on OLEDs elucidates the promising advantages this technology provides by now. However, there is still much room for improvement. For example, high brightness for lighting purposes can be achieved only at fairly high current densities accompanied by a strong decrease in long-term stability. Unfortunately, the required high current density additionally results in a decrease of the external quantum efficiency (EQE) [6,7].

In terms of device physics, the external quantum efficiency $\eta_{\text {ext }}$ is a crucial parameter of an OLED and is given by [8]

\footnotetext{
*Sebastian.Wehrmeister@physik.uni-augsburg.de †Wolfgang.Bruetting@physik.uni-augsburg.de
}

$$
\eta_{\mathrm{ext}}=\gamma \eta_{r} q_{\mathrm{eff}}(q) \eta_{\mathrm{out}} .
$$

Herein, $\gamma$ represents the charge-carrier balance, which describes the ratio of electrons and holes able to participate in the generation of the excited-state of emitter molecules ("excitons"); ideally, this factor equals $1 . \eta_{r}$ describes the fraction of created excitons that is allowed to decay radiatively under emission of a photon, which is determined by quantum mechanical selection rules. Because of strong spin-orbit coupling present in molecules comprising heavy-metal atoms like Pt or Ir, this factor is unity in phosphorescent OLEDs. $q_{\text {eff }}(q)$ defines the effective radiative quantum efficiency of the spin-allowed excited states. Therein, $q$ (often denoted as RQE) is called the intrinsic radiative quantum efficiency of the used guest-host system (a detailed explanation is given later), which is strongly influenced by optical microcavity effects summarized in the Purcell factor $F[9,10]$. Finally, $\eta_{\text {out }}$ characterizes the light outcoupling efficiency that is mainly affected by the optical constants and thicknesses of all layers of the device as well as by the orientation of the transition dipole moments of the emitting system under investigation. It gives a measure for the fraction of the internal converted power that is extracted to the outside world of an OLED. It was recently demonstrated that nonisotropic emitter orientation, as expressed by an anisotropy factor [11], has a significant impact on the 
external quantum efficiency of OLEDs and on the validity of efficiency analysis in terms of determining the factors listed in Eq. (1) $[12,13]$.

With respect to the expression for the EQE presented in Eq. (1), mainly, two sources for the above-mentioned efficiency roll-off at high current densities can be identified: First, a charge-carrier imbalance, e.g., due to different charge-carrier conductivity in the transport layers and inappropriate blocking layers. Second, exciton quenching processes reducing the effective RQE of the phosphorescent guest-host system. Changes of the light outcoupling factor induced by a shift of the emission zone may also play a role; however, due to the typically very thin emission layer $(10-20 \mathrm{~nm})$, the impact is less important. Two different mechanisms have been reported to influence the effective RQE, increasing the nonradiative decay rates: triplet-triplet annihilation (TTA) [14-16] and tripletpolaron quenching (TPQ) [17]. Again, a current-induced shift of the emission zone and therewith changes of the Purcell factor $F$ influencing the radiative decay rate are believed to play a minor role in this context. In the past, analyses on various matrix-emitter systems have assigned the EQE roll-off to TTA only, TPQ only, and a superposition of TTA and TPQ [17-21]. However, these investigations focused only on current densities higher than $1 \mathrm{~mA} / \mathrm{cm}^{2}$ disregarding quenching effects or carrier imbalances that occur already at much lower values of $j$.

In this article, we identify the quenching processes dominating the efficiency roll-off of red phosphorescent OLEDs with iridium(III)-bis(2-methyldibenzo-[f,h]quinoxaline)-(acetylacetonate) $\left[\operatorname{Ir}(\mathrm{MDQ})_{2}(\mathrm{acac})\right]$ as emitting molecule. In the first step, we compare the efficiency roll-off for direct emission calculated from current-voltageluminance $(I-V-L)$ characteristics to previously published results on the same matrix-emitter combination [22], pointing to TTA as the dominating quenching process in this system. However, additionally performed timeresolved experiments reveal that TPQ has a major impact on the excitonic lifetime up to a current density of $100 \mathrm{~mA} / \mathrm{cm}^{2}$ and, hence, on the resulting EQE. We discuss the origin of these apparently contradicting results from both experiments and show that TPQ can be identified as the dominant exciton quenching mechanism if radiative exciton lifetimes measured under extremely low driving conditions are considered. Furthermore, we demonstrate that a comprehensive description of the variation of the excited-state lifetimes with device thickness and current density is possible, using the aforedetermined parameters for radiative and nonradiative exciton decay.

\section{RESULTS AND DISCUSSION}

The stack layout in combination with three exemplary $I-V-L$ characteristics of the investigated bottom-emitting OLEDs is shown in Fig. 1. For this stack, indium tin oxide (ITO) is used as a transparent anode on top of a glass

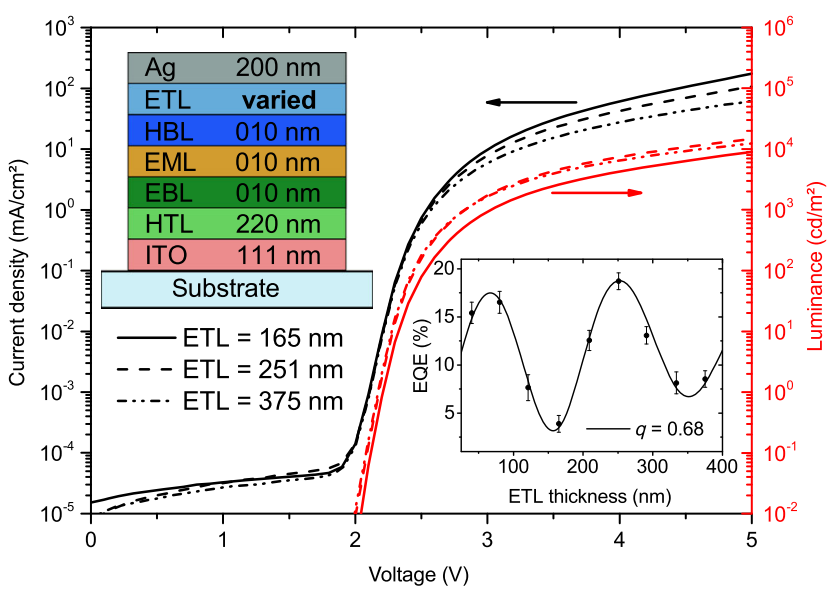

FIG. 1. Device stack layout with varying ETL thicknesses and exemplary current-voltage-luminance characteristics of the investigated OLEDs with ETL thicknesses of 165, 251, and $375 \mathrm{~nm}$. The inset in the lower right corner shows the measured $\mathrm{EQE}$ values for direct emission of all examined devices at a current density of $1 \mathrm{~mA} / \mathrm{cm}^{2}$ (dots) together with a fit based on numerical simulation (solid line) resulting in $q=0.68$ (see, also, Refs. [12,26]).

substrate, followed by thermally evaporated hole transport (HTL) and electron-blocking layers (EBLs). The emission layer (EML) of the investigated device consists of a red phosphorescent emitter $\operatorname{Ir}(\mathrm{MDQ})_{2}$ (acac) doped with a concentration of 8 wt \% in an $N, N^{\prime}$-bis(naphthalen-1yl)- $N, N^{\prime}$-bis(phenyl)benzidine ( $\alpha$-NPD) matrix. The top side of the stack is composed of a hole-blocking (HBL) and an electron transport layer (ETL) and is completed by a thick silver cathode. Materials of the HBL and EBL are NET-5 (Novaled GmbH, Germany) and $\alpha$-NPD, respectively. Both transport layers in these OLEDs are conductivity doped to improve the charge-carrier transport to the emission layer. Typically, these layers consist of a matrix material being identical to the neighboring blocking layers $[23,24]$ and dopants like metal oxides (e.g., $\mathrm{WO}_{3}, \mathrm{~V}_{2} \mathrm{O}_{5}$, or $\mathrm{MoO}_{3}$ ) for HTLs or alkali-metal salts (e.g., $\mathrm{Cs}_{2} \mathrm{CO}_{3}$ or $\mathrm{Cs}_{3} \mathrm{PO}_{4}$ ) for ETLs, respectively. Although the particular dopants used in our devices cannot be disclosed, we want to emphasize that essentially the same electrical characteristics are achieved with commercial dopants as published by Diez et al. [25]. We also want to note that Murawski et al. [22], who used yet another set of dopants, have reported almost identical $I-V$ characteristics and EQE data for their devices with an analogous ETL thickness variation.

Within the given stack layout, the doped transport layers act as optical spacers but can be considered as highly electrically conducting [27,28] (see the Supplemental Material [29] and Ref. [30]). Variations of the ETL thickness, therefore, change only the optical properties of the devices like the length of the microcavity. This circumstance affects the excitonic lifetime, the effective RQE, and the emission spectrum of the guest-host system embedded 
in the microcavity. Based on this stack layout, nine devices with varying ETL thicknesses between 40 and $375 \mathrm{~nm}$ are investigated. Further information on the fabrication process of these devices is given in Ref. [31]. The inset in the lower right corner of Fig. 1 displays the measured EQE values for direct emission, i.e., the light emission to air in the absence of any outcoupling structures, of all examined devices at a current density of $1 \mathrm{~mA} / \mathrm{cm}^{2}$. As explained in Ref. [26], optical simulations yield a radiative quantum efficiency $q=0.68 \pm 0.02$ of the investigated emitting system.

\section{A. External quantum efficiency roll-off}

By definition, the external quantum efficiency can be obtained by calculating the ratio of extracted photons and injected charges. To measure the total light emission, the angular dependence of the radiated light must be taken into account. Thus, the EQE is generally determined with an integrating sphere or a goniometer [32]. Here, the OLEDs are investigated in forward direction, and the ratio of luminance and applied current density is normalized to the measurements in a calibrated integrating sphere (see Fig. 1).

To identify the quenching process responsible for the efficiency roll-off, the data of the probed devices are fitted with relations for the two most probable quenching mechanisms. Based on the theory for TTA presented by Baldo et al. [17], the quantum efficiency of light emission can be written as

$$
\eta_{\text {ext }}(j)=\eta_{\text {ext }, 0} \frac{j_{0}}{4 j}\left(\sqrt{1+8 \frac{j}{j_{0}}}-1\right) .
$$

Here, $\eta_{\text {ext }, 0}$ describes the external quantum efficiency in the absence of TTA, i.e., at $j=0 \mathrm{~mA} / \mathrm{cm}^{2}$. Typically, EQE values measured at sufficiently low current densities, where quenching can be excluded, are chosen for this constant. The second parameter in this equation is called the critical current density $j_{0}$, which gives the current density at which the external quantum efficiency drops to half its initial value $\left[\eta_{\text {ext }}\left(j_{0}\right)=\eta_{\text {ext }, 0} / 2\right]$. Furthermore, the critical current density can be written as

$$
j_{0}=\frac{4 e d}{\Gamma_{\mathrm{TTA}} \tau^{2}} .
$$

Here, the parameters $e, d$, and $\tau$ are the electron charge, the thickness of the exciton formation zone (i.e., emission zone), and the excitonic lifetime. The fourth parameter $\Gamma_{\text {TTA }}$ is the decay rate for the nonradiative triplet-triplet annihilation. Despite its importance to characterize the efficiency roll-off, a direct measurement of $j_{0}$ is not advisable, as an electrical operation at such high current densities might lead to degradation [33]. In this context, it is worth mentioning that none of the devices show indications of degradation due to the performed investigations; i.e., all OLEDs fully recovered after the experiments.

An analogous approach by Reineke et al. [18] to describe the efficiency drop via TPQ results in

$$
\eta_{\mathrm{ext}}(j)=\frac{\eta_{\mathrm{ext}, 0}}{1+\tau C \Gamma_{\mathrm{TPQ}} j^{1 /(l+1)}} .
$$

The parameters $l$ and $C$ in this equation are defined through space-charge-limited current theory and can be written as [34,35]

$$
\begin{aligned}
C= & {\left[\left(\frac{l+1}{2 l+1}\right)\left(\frac{l+1}{l}\right)^{l}\left(\frac{N_{t}^{l} \epsilon}{d e^{2} \mu N_{c}}\right)\right]^{1 /(l+1)}, } \\
& \text { with } \quad l=E_{t} / k T .
\end{aligned}
$$

Here, $N_{c}$ and $N_{t}$ are the density of states at the transport level (here, the matrix material of the EML) and the total density of trap states (here, the phosphorescent dye in the EML), respectively. $E_{t}$ is the characteristic trap energy and describes the depth of trap states (here, the energy levels of the dye relative to the matrix). The elementary charge $e$, the mobility $\mu$, and the dielectric constant $\epsilon$ also appear in this equation. The parameter $d$ represents the thickness of the space charge zone in the investigated devices. With both transport layers of these OLEDs being conductivity doped, we assign this parameter to the EML plus probably a small fraction of both blocking layers (see the Supplemental Material [29]). To simplify the analysis, via a fitting process we introduce the single parameter $\xi=C \Gamma_{\mathrm{TPQ}} \cdot \tau$ represents the exited-state lifetime and can be measured via timeresolved spectroscopy as used and explained in detail later. Thus, Eq. (4) has only two unknown variables left, namely, $\xi$ and $l$. However, the simple Mott-Gourney relation $l=1$, i.e., the trap-free case, yields the best fit for all devices and was, therefore, used as a fixed parameter. Note that under these conditions, Eq. (4) corresponds to the expression given by Coehoorn et al. [21] if a constant mobility within the device is taken.

In Fig. 2, the results of both fits based on Eqs. (2) and (4) are shown along with the measured external quantum efficiencies. To characterize the efficiency roll-off, only data for current densities higher than $j=10^{-1} \mathrm{~mA} / \mathrm{cm}^{2}$ are taken into account. For both fits, the external quantum efficiency at $j=0.59 \mathrm{~mA} / \mathrm{cm}^{2}$ is chosen as $\eta_{\text {ext }, 0}$. It can clearly be seen from this figure that the fit based on TPQ as the dominant quenching process cannot describe the progression in an acceptable way, whereas the TTA fit is in very good agreement with the experimental data set for $j>0.5 \mathrm{~mA} / \mathrm{cm}^{2}$. With $j_{0}=148 \pm 1 \mathrm{~mA} / \mathrm{cm}^{2}$, this result is at the lower boundary of the values presented in Ref. [22]. Thus, the TTA fit also confirms a direct comparability of our device data to Murawski et al. [22]. In respect thereof, the assumption of a TTA-based quenching process responsible for the efficiency roll-off in 
these devices seems comprehensible. However, since most investigations focus only on current densities higher than $10^{-1} \mathrm{~mA} / \mathrm{cm}^{2}$, a huge disadvantage of this method is often overlooked. With Fig. 2 illustrating a wider range of $j$, the significant drop of EQE at lower current densities must be subject to discussions as well. With respect to Eq. (1), this behavior can be explained only with a nonconstant chargecarrier balance. In detail, we ascribe the drop of the EQE at small current densities (below $j \approx 10^{-1} \mathrm{~mA} / \mathrm{cm}^{2}$ ) to an imbalance of $\gamma$ due to the preferred hole transport for $\alpha$ NPD [36]. Thus, the presented experimental data in Fig. 2 is subject to changes of $\gamma$ as well as of $q_{\text {eff }}$. A general analysis of the EQE with Eqs. (2) and (4) is, therefore, not reliable because it is impossible to tell at which current densities the efficiency roll-off is exclusively affected by variations of $q_{\text {eff }}$. In respect to this conclusion, a more profound investigation of $q_{\text {eff }}$ seems necessary.

\section{B. Time-resolved spectroscopy}

To further investigate the efficiency roll-off for this OLED stack in more detail, we perform time-resolved photoluminescence spectroscopy in dependence of the current density through the device. Conceptually, we follow the route presented by Giebink and Forrest [37]. In addition to a rectangular electrical pulse applied to the OLED, a stimulation to photoluminescence (PL) with a short laser pulse on top of the quasi-steady-state electroluminescence (EL) is

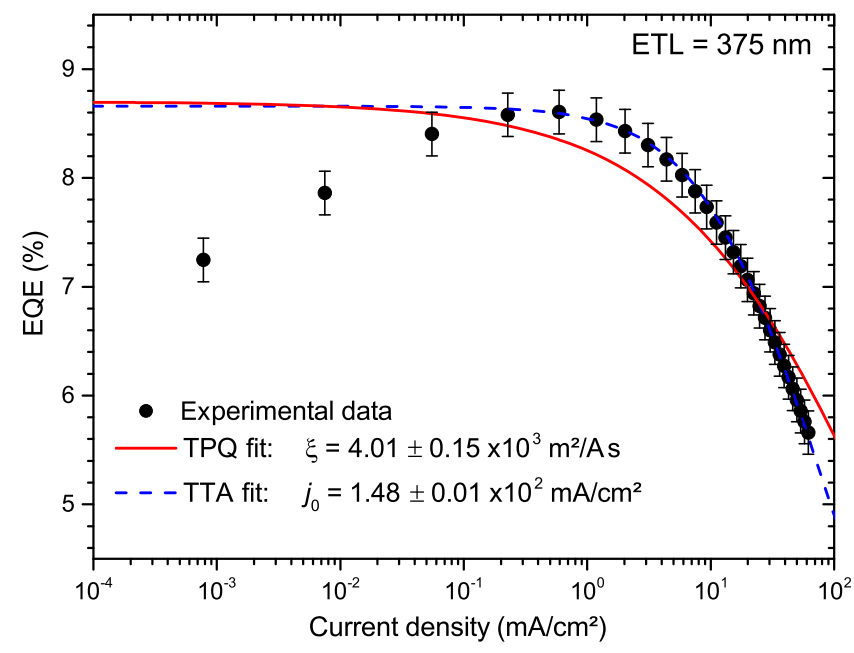

FIG. 2. External quantum efficiency as a function of the applied current density (dots). The error bars represent an upper estimation of an error margin with $0.2 \%$ (absolute). The data points can be fitted with Eq. (2) (dashed blue line), assuming TTA is the only quenching process present. A second fit based on Eq. (4) (solid red line) is performed presuming TPQ as the dominant process. Following Ref. [22], only EQE data for current densities higher than $j=10^{-1} \mathrm{~mA} / \mathrm{cm}^{2}$ are taken into account. The TTA fit describes the presented information very well yielding $j_{0}=$ $148 \mathrm{~mA} / \mathrm{cm}^{2}$ as the measure for the efficiency roll-off. performed. A schematic illustration of the used pulse sequence is shown in Fig. 3. By analyzing the decay time of the PL signal as a function of the applied current density, the excitonic lifetime and its modification by quenching processes can be identified. In contrast to the EQE, variations of $\gamma$ have no impact on the effective RQE as it is affected only by changes of the excitonic lifetimes (a detailed explanation is given later), granting this method a significant advantage over the analysis presented in Fig. 2.

The electrical excitation is achieved by an arbitrary pulse generator from Tabor Electronics (WW2571A). To ensure steady-state conditions, a pulse length of $25 \mu$ s is chosen. The optical excitation of the emission layer is performed with a nitrogen laser from Laser Technik Berlin (MNL202C, wavelength $337.1 \mathrm{~nm}$, pulse energy approximately $1 \mu \mathrm{J}$, duration $750 \mathrm{ps}$ ), and it is ensured that the excitation is located only on the active area of the device. The emitted light is collected in the direction of the surface normal, and a temporal and spectral analysis is performed using a streak-camera system (C5680, Hamamatsu) combined with a spectrograph (SpectraPro 2300i, Princeton Instruments).

Integration over the emission spectrum results in the intensity decay as a function of time under different applied voltages and the resulting current density through the device. Figure 4 illustrates the temporal behavior of the photoluminescence decay for two different current densities. Both time traces can be described by monoexponential fit functions following the equation

$$
I(t)=I_{0} \exp \left[-\left(t / \tau^{*}\right)\right]+C
$$

The constants $I_{0}$ and $C$ represent the initial intensity of the emitted light at the end of the laser pulse and the

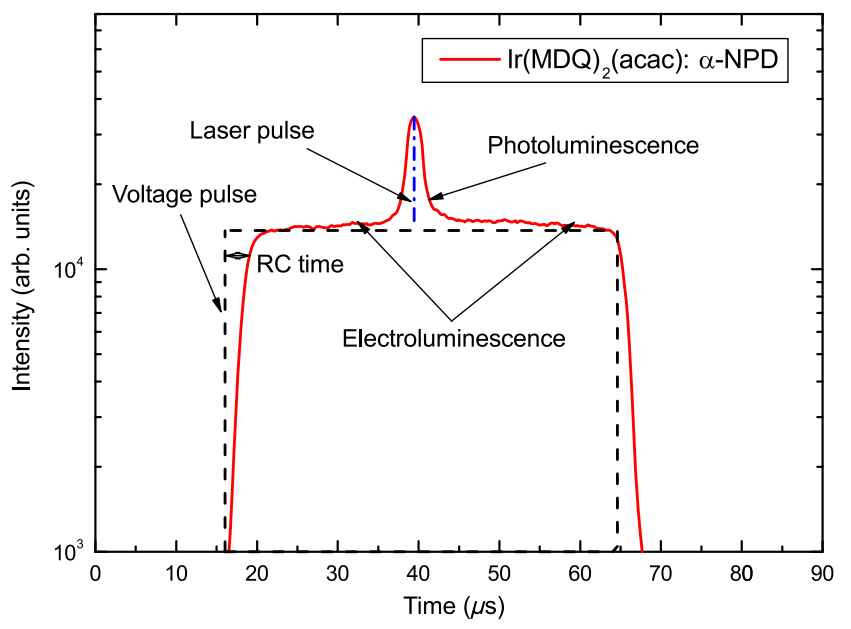

FIG. 3. Illustration of an exemplary pulse sequence performed on the red phosphorescent OLEDs. The dashed lines represent the electrical and optical excitation. The red solid line displays the measured luminescence as a reaction to both excitations. For better visual separation of the EL and PL, an electrical pulse duration of $50 \mu \mathrm{s}$ is chosen for this illustration. 
background intensity due to the electrical driving and noise, respectively. The parameter $\tau^{*}$ represents the excitonic lifetime at the given driving conditions and is the main parameter in the fitting process.

In the absence of any cavity effects (e.g., in an infinite bulk of the emitter material) and for low excitation densities, the intrinsic excited-state lifetime is defined as

$$
\tau_{0}=\left(\Gamma_{\mathrm{r}}+\Gamma_{\mathrm{nr}}\right)^{-1} .
$$

Therein, $\Gamma_{\mathrm{r}}$ and $\Gamma_{\mathrm{nr}}$ express the (intrinsic) radiative and nonradiative decay rates, respectively. All three parameters in Eq. (7) can be accessed with time-resolved spectroscopy [11-13] and have been previously determined for the devices under investigation [26]. As already mentioned, if the emitting molecules are embedded in a microcavitylike structure, such as an OLED, the so-called Purcell effect must be taken into account [9]. Thus, the radiative decay rate is modified by the Purcell factor $F$ due to, e.g., interference effects, while the nonradiative decay rate stays unaffected by the microcavity [38]. Equation (7), therefore, changes to

$$
\tau=\left(F \Gamma_{\mathrm{r}}+\Gamma_{\mathrm{nr}}\right)^{-1}
$$

With rising current density, additional induced nonradiative decay channels such as TTA and TPQ become more relevant. To incorporate such effects, $\Gamma_{\mathrm{nr}}^{*}(j)$ represents the modified nonradiative decay rate as a function of the current density. In detail, it can be written as a superposition of the intrinsic nonradiative decay rate $\Gamma_{\mathrm{nr}}$ in the absence of current flow and a current-induced nonradiative decay rate $\Gamma_{\mathrm{nr}}(j)$. With all earlier-mentioned influences taken into account, the experimentally measured excited-state lifetime in a working OLED is then given by

$$
\tau^{*}=\left[F \Gamma_{\mathrm{r}}+\Gamma_{\mathrm{nr}}^{*}(j)\right]^{-1} .
$$

Figure 4 illustrates that the measured photoluminescence decay for a current density of $62.5 \mathrm{~mA} / \mathrm{cm}^{2}$ is faster than without current flow. With regard to Eq. (9), this observation corresponds to a shorter lifetime $\tau^{*}$ of $1.08 \mu$ s as compared to $\tau=1.45 \mu \mathrm{s}$ in the absence of current and, thus, a higher nonradiative decay rate $\Gamma_{\mathrm{nr}}^{*}(j)$. Consequently, a decrease of the RQE with increasing $j$ can be expected. Based on this observation, a systematic study of the excitonic lifetime with rising current densities is performed. In Fig. 5, the determined excited-state lifetimes are displayed as a function of the current density. Since the PL lifetime $\tau$ without current flow cannot be displayed on a logarithmic scale for the current density, the respective data point is plotted as a gray hatched horizontal bar (the thickness resembles the error range). The observed data show a rapid decrease until a value of $\tau^{*}=0.99 \mu \mathrm{s}$ is reached at $100 \mathrm{~mA} / \mathrm{cm}^{2}$, representing a drop of more than $30 \%$ from the initial value. As all of the data points shown in Fig. 5 are determined by monoexponential fits based on Eq. (6), it

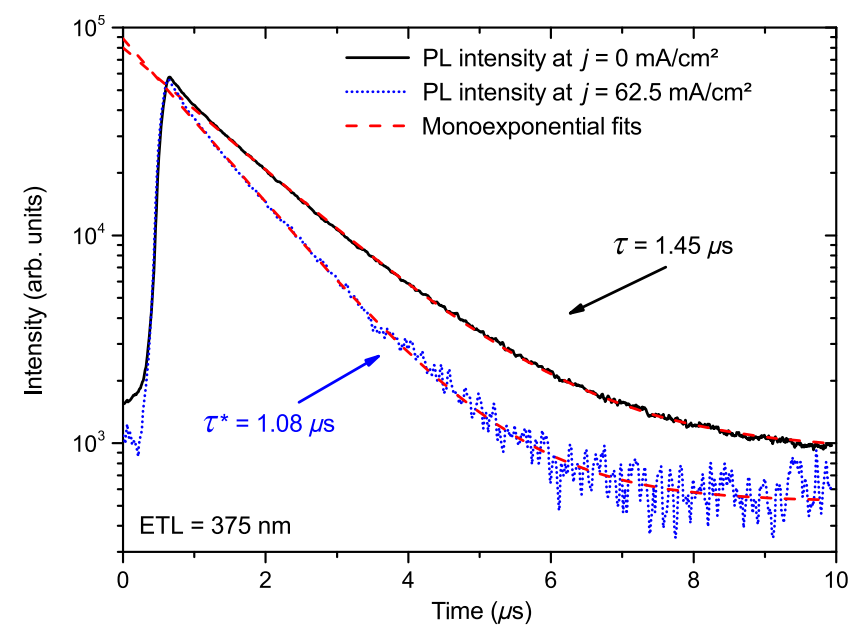

FIG. 4. Comparison of the time-resolved photoluminescence transient without (solid black line) and with an electrical pulse (dotted blue line). Dashed red lines represent monoexponential fits according to Eq. (6). An analysis of the excited-state lifetimes shows a current-induced reduction of $\tau$ due to increased nonradiative decay rates.

seems unlikely that TTA is the underlying process in this device because this effect would require a biexponential fit due to the bimolecular nature of the mechanism [37].

Thus, a TPQ-dominated quenching process seems probable. To support this statement, the decrease of the excitonic lifetimes as a function of the current density is fitted by

$\tau^{*}=\frac{1}{F \Gamma_{\mathrm{r}}+\left(\Gamma_{\mathrm{nr}}+\xi j^{1 /(l+1)}\right)} \Rightarrow \frac{1}{F \Gamma_{\mathrm{r}}+\left(\Gamma_{\mathrm{nr}}+\xi j^{1 / 2}\right)}$.

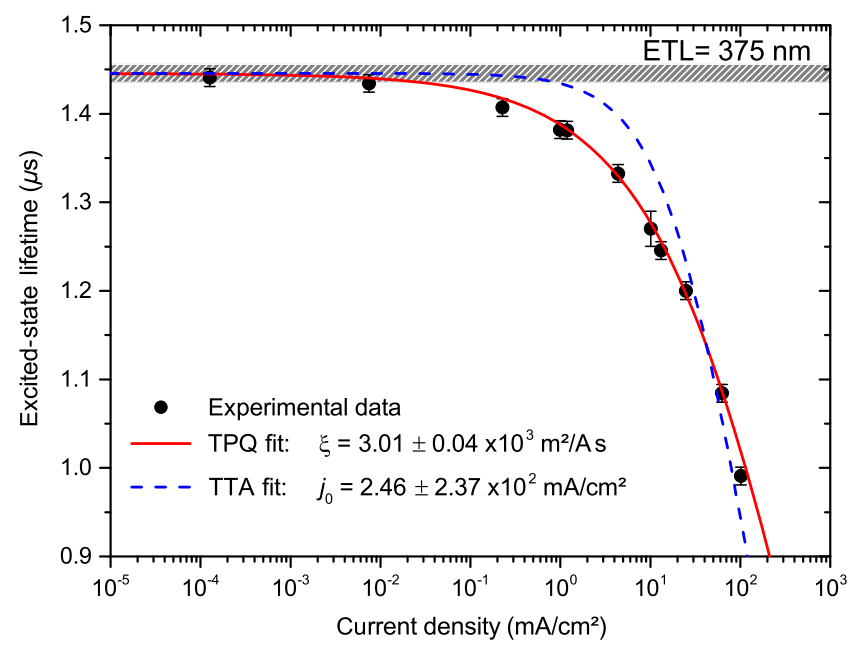

FIG. 5. Excited-state lifetimes in dependence of the applied current density during the EL-PL experiments described in Fig. 3. A comparison of the TPQ fit (solid red line) with the TTA fit (dashed blue line) based on Eqs. (10) and (11), respectively, stands in great contrast to previously drawn conclusions. Both fits are normalized to the initial value of $\tau^{*}$ (gray hatched beam) obtained through PL-only measurements. 
This relation is a direct conclusion of the formulas on tripletpolaron quenching presented in Ref. [18]. Since the parameter $l$ is fixed to the Mott-Gourney case, Eq. (10) can be further simplified setting $l=1$. With the relation for the effective quantum efficiency $q_{\text {eff }}=F \Gamma_{\mathrm{r}} \tau$ and the assumption that all other factors in Eq. (1) stay constant for different driving conditions, a similar approach for TTA [17] based on Eq. (2) yields

$$
\tau^{*}=\tau \frac{j_{0}}{4 j}\left(\sqrt{1+8 \frac{j}{j_{0}}}-1\right) .
$$

In Fig. 5, both fits are shown alongside the experimental lifetime data set achieved by time-resolved optical spectroscopy. Reassuring the earlier-mentioned hypothesis of a TPQ-dominated quenching process responsible for the drop of the excited-state lifetimes, the corresponding fit based on Eq. (10) describes the measured data very well. For the normalization to the initial value of the PL lifetime without current flow, the parameters $\Gamma_{\mathrm{r}}=(5.1 \pm 0.1) \times 10^{5} \mathrm{~s}^{-1}$, $\Gamma_{\mathrm{nr}}=(2.2 \pm 0.2) \times 10^{5} \mathrm{~s}^{-1}$ (taken from Ref. [26]) are chosen. In comparison to Fig. 2, the absolute value as well as the error margin of the TPQ fit parameter is reduced to $\xi=(3.01 \pm 0.04) \times 10^{3} \mathrm{~m}^{2} / \mathrm{As}$. The analogous analysis with the TTA fit based on Eq. (11) shows a significant discrepancy to the experimental data. Especially in the region between $10^{-1}$ and $10^{1} \mathrm{~mA} / \mathrm{cm}^{2}$, the TTA fit highly underestimates the drop of the excited-state lifetimes. The great difficulties the fit has describing the progression of the data set is also easily visible in the resulting fit parameter $j_{0}=2.46 \pm 2.37 \mathrm{~mA} / \mathrm{cm}^{2}$ with its error margin of nearly $100 \%$.

From the literature, it is known that TTA highly depends on the resulting exciton density within the emission layer [15]. Therefore, we estimate triplet exciton densities for both types of excitations. Here, we follow the route of Baldo et al. [17]. For electrical excitation, the calculations yield exciton densities between $10^{12}$ and $10^{18} \mathrm{~cm}^{-3}$ depending on the current density. The upper limit for the laser excitation in this experiment, i.e., if all excitons formed on the matrix are transferred to the emitter, is calculated with the absorption coefficient for $\alpha$-NPD [39] of $1.5 \times 10^{5} \mathrm{~cm}^{-1}$ and corresponds to a triplet exciton density of $10^{17} \mathrm{~cm}^{-3}$. Even though TTA has been reported for excitation densities of the same order of magnitude, this observation does not allow for a generalization, as TTA is highly device dependent [18].

With similar results being seen in all nine devices with different ETL thicknesses (see the Supplemental Material [29]), a TTA-dominated quenching process can be ruled out up to a current density of at least $100 \mathrm{~mA} / \mathrm{cm}^{2}$ based on time-resolved spectroscopy. On the other hand, the progression of the excited-state lifetimes can be well described with TPQ for all ETL thicknesses. Therefore, these experiments clearly support the predictions for a TPQ-dominated quenching mechanism in phosphorescent OLEDs based on Monte Carlo studies by van Eersel et al. [20]. However, this result is in great contrast to the conclusions drawn from Fig. 2 and earlier published results in Ref. [22]. Note that Murawski et al. [22] used different transport materials in their devices, which might lead to differences in the internal carrier distributions.

\section{Comparison of both analyses}

To further analyze the disagreement between the EQEbased and the lifetime-based quenching analysis, a different approach seems helpful disentangling the factors in Eq. (1). Based on the definition of the intrinsic radiative quantum efficiency of an emitting system $q=\Gamma_{\mathrm{r}} \tau_{0}$, the effective $\mathrm{RQE}$ as a function of the current density can be written as

$$
q_{\mathrm{eff}}(j)=\frac{F \Gamma_{\mathrm{r}}}{F \Gamma_{\mathrm{r}}+\Gamma_{\mathrm{nr}}^{*}(j)}=F \Gamma_{\mathrm{r}} \tau^{*} .
$$

In Fig. 6, the normalized effective RQE $=q_{\text {eff }}(j) / q_{\text {eff }}(0)$ calculated from the measured excited-state lifetimes using $\Gamma_{\mathrm{r}}=5.1 \times 10^{5} \mathrm{~s}^{-1}$ (taken from Ref. [26]) is plotted versus the applied current density. The normalized TTA and TPQ fits from Fig. 5 [see Eqs. (11) and (10)] are presented as well. For comparison, the directly measured EQE data and the TTA fit from Fig. 2 are also plotted. Both the directly measured EQE and the corresponding TTA fit are scaled to match the normalized effective RQE at $j=1 \mathrm{~mA} / \mathrm{cm}^{2}$. As already mentioned in connection with Fig. 2, the progression of the directly measured $\mathrm{EQE}$ is a superposition of changes in the effective RQE as well as of the charge-carrier balance factor $\gamma$. At low current densities $j<10^{-1} \mathrm{~mA} / \mathrm{cm}^{2}$, the EQE is still increasing as the

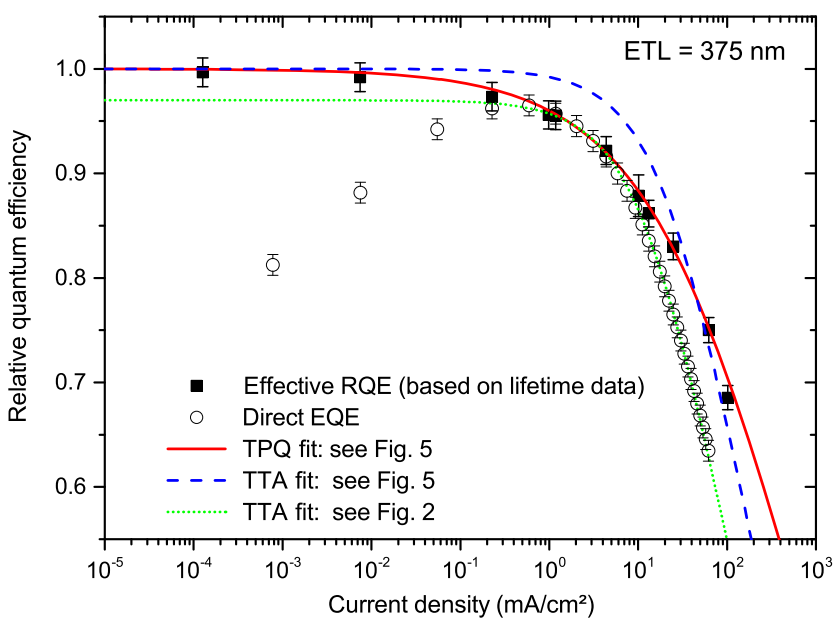

FIG. 6. Comparison of the calculated relative effective RQE (square) with the normalized measured EQE (circle). For clarity, the plot with $j_{0}=148 \pm 1 \mathrm{~mA} / \mathrm{cm}^{2}$ (dotted green line) from Fig. 2 is presented in addition to the measured data set. The TPQ (solid red line) and TTA fit (dashed blue line) from Fig. 5 are normalized as well to emphasize the differences of both methods. 
charge-carrier balance is not yet perfect. The $\mathrm{EQE}$ reaches its maximum at about $0.6 \mathrm{~mA} / \mathrm{cm}^{2}$; however, at this current, the RQE has already dropped by almost $5 \%$. Thus, taking the EQE value at this maximum as $\eta_{\text {ext, } 0}$ for the TTA-based analysis clearly underestimates the true value.

Moreover, at current densities higher than $10 \mathrm{~mA} / \mathrm{cm}^{2}$, an additional discrepancy of the direct $\mathrm{EQE}$ to the calculated effective RQE determined from lifetime data is visible. For clarity, a different illustration is presented in Fig. 7. Here, the EQE obtained from $I-V-L$ characteristics (e.g., see Fig. 2) is shown alongside the effective RQE based on the measured excited-state lifetimes for three different ETL thicknesses. For the latter, $q_{\text {eff }}$ from Eq. (12) is multiplied by a constant factor (the outcoupling efficiency of the devices) to normalize the data sets to the $\mathrm{EQE}$ at $j=1 \mathrm{~mA} / \mathrm{cm}^{2}$ (see inset of Fig. 1). From Fig. 7 it is clearly visible that both methods for obtaining the EQE match well with each other up to current densities of $10 \mathrm{~mA} / \mathrm{cm}^{2}$. However, at higher current densities, deviations become obvious, where the $I-V-L$ data sets fall below the effective RQE from the excited-state lifetimes. From previous studies, it is known that electron transport in $\alpha$-NPD: $\operatorname{Ir}(\mathrm{MDQ})_{2}(\mathrm{acac})$ takes place via hopping between emitter molecules [40]. In combination with Eq. (1), the deviations for current densities higher than $10^{1} \mathrm{~mA} / \mathrm{cm}^{2}$ can probably be explained by a reduction of $\gamma$ after reaching an electron transportation limit.

In summary, Figs. 6 and 7 highlight the most important aspects for analyzing quenching mechanisms. From all the presented data, it becomes clear that the analysis based on direct $\mathrm{EQE}$ measurements is insufficient to fully characterize the efficiency roll-off in OLEDs. Likewise, to the presented investigations, a fit of the EQE roll-off (see Fig. 2) might mislead the observer because quenching occurring already

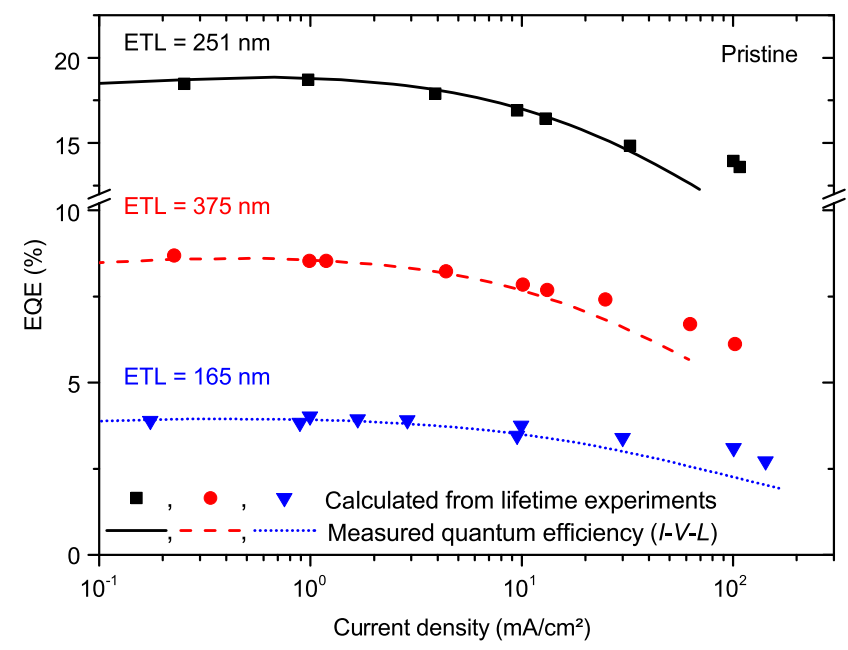

FIG. 7. External quantum efficiency obtained through $I-V-L$ characteristics in comparison with calculations based on timeresolved measurements for three different ETL thicknesses. For clarity, all data are shown without error bars. at very low current densities leads to an erroneous initialization parameter $\eta_{\mathrm{ext}, 0}$ and any changes of $\gamma$ further affecting the efficiency stay undetected. Here, timeresolved spectroscopic experiments (see Fig. 3) reveal TPQ as the responsible quenching mechanism based on the fact that the excitonic lifetimes are independent from $\gamma$. Furthermore, this method allows an investigation even at very low current densities revealing the influence of quenching on the excitonic lifetimes over a wide range of $j$.

\section{Device-specific parameters for TPQ}

With the transport layers being conductivity doped, major influences of the ETL thickness on the quenching behavior of the devices can be neglected. Thus, in combination with identical emission layers, all nine devices comprising different ETL thicknesses are expected to show similar relative quenching. Taking the arithmetic mean of the parameters obtained through the TPQ fits for all ETL thicknesses (see Table I and the Supplemental Material [29]), a global parameter $\bar{\xi}=(2.81 \pm 0.17) \times 10^{3} \mathrm{~m}^{2} / \mathrm{A} \mathrm{s}$ for this OLED stack can be obtained. For the error margin, the standard deviation is chosen. As mentioned before, the simple Mott-Gourney case $l=1$ is used for all fits.

With the definition of $\xi$ and Eq. (5), we are able to calculate the triplet-polaron quenching rate $\Gamma_{\mathrm{TPQ}}$ (see Table I) yielding an average of $\overline{\Gamma_{\mathrm{TPQ}}}=(2.90 \pm 0.18) \times$ $10^{-11} \mathrm{~cm}^{3} / \mathrm{s}$ for all nine devices. Since we do not measure transport properties for each material in this stack, we follow the route of Reineke et al. [18] using the same assumptions. As compared to Reineke et al. [18], who observed quenching rates of $(2-7) \times 10^{-13} \mathrm{~cm}^{3} / \mathrm{s}$, our values are 2 orders of magnitude higher. However, this difference is not very surprising, as they investigated unipolar devices that showed $l=2$. This value suggests that charges are being trapped, most likely on the phosphorescent dye. Although trapped charges will also

TABLE I. Based on the fit parameter $\xi$ and the assumptions made by Reineke et al. [18], the triplet-polaron quenching rate $\Gamma_{\mathrm{TPQ}}$ can be calculated for each device. With these values, the arithmetic mean and standard deviation $\bar{\xi}=(2.81 \pm$ $0.17) \times 10^{3} \mathrm{~m}^{2} / \mathrm{As}$ and $\overline{\Gamma_{\mathrm{TPQ}}}=(2.90 \pm 0.18) \times 10^{-11} \mathrm{~cm}^{3} / \mathrm{s}$ are calculated.

\begin{tabular}{lcc}
\hline \hline EL $(\mathrm{nm})$ & $\xi\left(10^{3} \mathrm{~m}^{2} / \mathrm{As}\right)$ & $\Gamma_{\mathrm{TPQ}}\left(10^{-11} \mathrm{~cm}^{3} / \mathrm{s}\right)$ \\
\hline 40 & $3.65 \pm 0.22$ & $3.77 \pm 0.23$ \\
80 & $2.85 \pm 0.02$ & $2.95 \pm 0.02$ \\
121 & $2.63 \pm 0.16$ & $2.72 \pm 0.16$ \\
165 & $2.80 \pm 0.21$ & $2.89 \pm 0.22$ \\
209 & $2.27 \pm 0.12$ & $2.34 \pm 0.12$ \\
251 & $3.16 \pm 0.12$ & $3.27 \pm 0.12$ \\
291 & $1.96 \pm 0.63$ & $2.02 \pm 0.65$ \\
334 & $2.93 \pm 0.11$ & $3.03 \pm 0.14$ \\
375 & $3.00 \pm 0.04$ & $3.10 \pm 0.04$ \\
\hline \hline
\end{tabular}


contribute to TPQ, the rate constant might be different. On the other hand, quenching rates of $(5.0-7.5) \times$ $10^{-12} \mathrm{~cm}^{3} / \mathrm{s}$ in an OLED structure comprising a green phosphorescent Ir complex have been reported by Kalinowski et al. [41], which are comparable to our values within a factor of 2 . However, without detailed information on the recombination zone within the emissive layer, it is hard to compare data from different publications quantitatively. In our case, we assume that TPQ occurs over the complete width of the EML. Therefore, the values for $\Gamma_{\mathrm{TPQ}}$ from our calculations represent an upper limit. Furthermore, it is worth mentioning that TTA rates can be of the same order of magnitude in some devices $[17,18,33]$. This observation demonstrates that the dominant quenching mechanism is highly device dependent and has to be determined for each OLED anew.

With this parameter set, one should be able to describe the current-induced decrease of the excited-state lifetimes for all devices. In Fig. 8, the measured excited-state lifetimes for four applied current densities are plotted vs the ETL thickness (symbols). In addition, Eq. (10) is used to calculate the expected excitonic lifetimes based on a single value of $\bar{\xi}=(2.81 \pm 0.17) \times 10^{3} \mathrm{~m}^{2} / \mathrm{As}$ (lines). For all four current densities, the calculation is in very good agreement with the measurements even with the somewhat larger spreading at $j=100 \mathrm{~mA} / \mathrm{cm}^{2}$, which we ascribe to high stress levels, i.e., temperature increase or parasitic voltage drops at the contacts.

Note that the magnitude of the decrease of the excitonic lifetime by TPQ is different for different ETL thicknesses. Yet, this does not mean that the quenching process itself is cavity dependent. From Eq. (4), it is obvious that changes

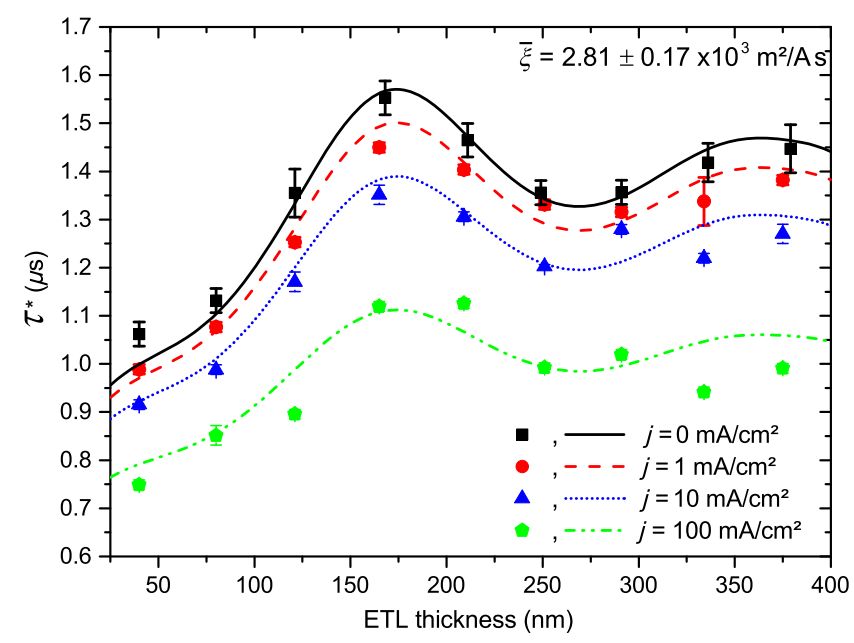

FIG. 8. Comparison of the measured excited-state lifetime (symbols) with calculations (lines) based on the parameter set $l=1$ and $\bar{\xi}=(2.81 \pm 0.17) \times 10^{3} \mathrm{~m}^{2} / \mathrm{As}$ for four current densities. With the measured data being in very good agreement with the calculations, the assumption of a cavity-independent quenching rate $\Gamma_{\mathrm{TPQ}}$ can be confirmed. of the excited-state lifetime due to cavity effects affect the overall magnitude of the EQE quenching as well. There is a higher probability for TPQ as the excitonic lifetime increases in an antinode of the OLED cavity. However, the overall trend in the variation of $\tau^{*}$ visible in Fig. 8 simply reflects the Purcell factor. From these results, we conclude that although the total magnitude of quenching is proportional to the excited-state lifetime, the relative quenching as expressed by a cavity-independent $\Gamma_{\mathrm{TPQ}}$ is the same in all devices. A similar result was recently obtained by Murawski et al. [22] assuming TTA as the dominant mechanism. In addition, Fig. 8 illustrates again very clearly that even at a relatively low current density of $j=1 \mathrm{~mA} / \mathrm{cm}^{2}$, quenching occurs, significantly reducing the excited-state lifetimes compared to the PL-only experiment.

\section{CONCLUSION}

In this article, we compare the conventional quenching analysis based on the EQE roll-off in phosphorescent OLEDs derived from $I-V-L$ characteristics to time-resolved spectroscopic measurements combining electrical and optical excitations. The latter experiment directly probes changes of the triplet lifetime due to current-induced quenching processes yielding the radiative quantum efficiency of the emitter material, while the former additionally depends on other factors like the charge-carrier balance in the device. Our data clearly show that quenching effects already occur at rather low current densities (much lower than $10^{-1} \mathrm{~mA} / \mathrm{cm}^{2}$ ), where the conventional $\mathrm{EQE}$ measurements are not reliable, probably due to imbalanced charge-carrier injection. Thus, an analysis based on EQE data suffers from an underestimation of the normalization parameter $\eta_{\mathrm{ext}, 0}$, which, in turn, can lead to the erroneous conclusion of a TTA-based quenching mechanism for the investigated guest-host system.

Instead, time-resolved spectroscopy probes the radiative quantum efficiency and, thus, gives direct access to exciton quenching processes even in the limit of vanishing current density. For the red phosphorescent emitter $\operatorname{Ir}(\mathrm{MDQ})_{2}$ (acac) doped into an $\alpha$-NPD matrix, triplet-polaron quenching gives an excellent description of the lifetime data over a wide range of current densities $\left(10^{-4}-10^{2} \mathrm{~mA} / \mathrm{cm}^{2}\right)$. Similar results have recently been obtained for two other phosphorescent emitters by van Eersel et al. [20]. Additionally, we present evidence that the TPQ quenching rate $\Gamma_{\mathrm{TPQ}}$ is independent from the OLED microcavity.

In conclusion, we present a method for a comprehensive analysis of the quenching processes in phosphorescent OLEDs. This method allows for a critical evaluation of both TPQ and TTA as potential origin for efficiency roll-off over a wide range of current densities. A comparison to the widespread but oversimplified analysis of the EQE reveals that the underlying procedure is very critical to proper normalization at low currents, which could explain controversial results found in the literature. 


\section{ACKNOWLEDGMENTS}

The authors would like to acknowledge financial support by the German Federal Ministry of Education and Research (BMBF) within the projects "TOPAS 2012" and "OLYMP" (Contracts No. FKZ 13N10474 and No. 13N12240). Additionally, we thank the Deutsche Forschungsgemeinschaft for financial support under the Contract No. BR 1728/13-1.

[1] C. W. Tang and S. A. VanSlyke, Organic electroluminescent diodes, Appl. Phys. Lett. 51, 913 (1987).

[2] C. W. Tang, S. A. VanSlyke, and C. H. Chen, Electroluminescence of doped organic thin films, J. Appl. Phys. 65, 3610 (1989).

[3] M. A. Baldo, D. F. O’Brien, Y. You, A. Shoustikov, S. Sibley, M. E. Thompson, and S. R. Forrest, Highly efficient phosphorescent emission from organic electroluminescent devices, Nature (London) 395, 151 (1998).

[4] Y. Kawamura, K. Goushi, J. Brooks, J. J. Brown, H. Sasabe, and C Adachi, 100\% phosphorescence quantum efficiency of ir(iii) complexes in organic semiconductor films, Appl. Phys. Lett. 86, 071104 (2005).

[5] M. Pfeiffer, K. Leo, X. Zhou, J. S. Huang, M. Hofmann, A. Werner, and J. Blochwitz-Nimoth, Doped organic semiconductors: Physics and application in light emitting diodes, Org. Electron. 4, 89 (2003).

[6] C. Adachi, M. A. Baldo, M. E. Thompson, and S. R. Forrest, Nearly $100 \%$ internal phosphorescence efficiency in an organic light-emitting, J. Appl. Phys. 90, 5048 (2001).

[7] N. C. Giebink, B. W. D'Andrade, M. S. Weaver, P. B. Mackenzie, J. J. Brown, M. E. Thompson, and S. R. Forrest, Intrinsic luminance loss in phosphorescent small-molecule organic light emitting devices due to bimolecular annihilation reactions, J. Appl. Phys. 103, 044509 (2008).

[8] T. Tsutsui, E. Aminaka, C. P. Lin, and D.-U. Kim, Extended molecular design concept of molecular materials for electroluminescence: Sublimed-dye films, molecularly doped polymers and polymers with chromophores, Phil. Trans. R. Soc. A 90, 5048 (2001).

[9] E. M. Purcell, Spontaneous emission probabilities at radio frequencies, Phys. Rev. 69, 681 (1946).

[10] S. Nowy, N. A. Reinke, J. Frischeisen, and W. Brütting, Light extraction and optical loss mechanisms in organic light-emitting diodes, Proc. SPIE Int. Soc. Opt. Eng. 6999, 69992V (2008).

[11] T. D. Schmidt, B. J. Scholz, C. Mayr, and W. Brütting, Efficiency analysis of organic light-emitting diodes based on optical simulations, IEEE Sel. Top. Quantum Electron. 19, 1 (2013).

[12] T. D. Schmidt, D. S. Setz, M. Flämmich, J. Frischeisen, D. Michaelis, B. C. Krummacher, N. Danz, and W. Brütting, Evidence for non-isotropic emitter orientation in a red phosphorescent organic light-emitting diode and its implications for determining the emitter's radiative quantum efficiency, Appl. Phys. Lett. 99, 163302 (2011).

[13] T. D. Schmidt, D. S. Setz, M. Flämmich, J. Frischeisen, D. Michaelis, C. Mayr, A. F. Rausch, T. Wehlus, B. J. Scholz,
T. C. G. Reusch, N. Danz, and W. Brütting, Comprehensive efficiency analysis of organic light-emitting diodes featuring emitter orientation and triplet-to-singlet up-conversion, Appl. Phys. Lett. 103, 093303 (2013).

[14] W. Staroske, M. Pfeiffer, K. Leo, and M. Hoffmann, SingleStep Triplet-Triplet Annihilation: An Intrinsic Limit for the High Brightness Efficiency of Phosphorescent Organic Light Emitting Diodes, Phys. Rev. Lett. 98, 197402 (2007).

[15] C. Murawski, K. Leo, and M. C. Gather, Efficiency roll-off in organic light-emitting diodes, Adv. Mater. 25, 6801 (2013).

[16] S. M. King, M. Cass, M. Pintani, C. Coward, F. B. Dias, A. P. Monkman, and M. Roberts, The contribution of triplettriplet annihilation to the lifetime and efficiency of fluorescent polymer organic light emitting diodes, J. Appl. Phys. 109, 074502 (2011).

[17] M. A. Baldo, C. Adachi, and S. R. Forrest, Transient analysis of organic electrophosphorescence. ii. Transient analysis of triplet-triplet annihilation, Phys. Rev. B 62, 10967 (2000).

[18] S. Reineke, K. Walzer, and K. Leo, Triplet-exciton quenching in organic phosphorescent light-emitting diodes with IR-based emitters, Phys. Rev. B 75, 125328 (2007).

[19] D. Song, S. Zhao, Y. Luo, and H. Aziz, Causes of efficiency roll-off in phosphorescent organic light emitting devices: Triplet-triplet annihilation versus triplet-polaron quenching, Appl. Phys. Lett. 97, 243304 (2010).

[20] H. van Eersel, P. A. Bobbert, R. A. J. Janssen, and R. Coehoorn, Monte Carlo study of efficiency roll-off of phosphorescent organic light-emitting diodes: Evidence for dominant role of triplet-polaron quenching, Appl. Phys. Lett. 105, 143303 (2014).

[21] R. Coehoorn, H. van Eersel, P. Bobbert, and R. Janssen, Kinetic Monte Carlo study of the sensitivity of oled efficiency and lifetime to materials parameters, Adv. Funct. Mater., doi: (2014).

[22] C. Murawski, P. Liehm, K. Leo, and M. C. Gather, Influence of cavity thickness and emitter orientation on the efficiency roll-off of phosphorescent organic light-emitting diodes, Adv. Funct. Mater. 24, 1117 (2014).

[23] G. Schwartz, S. Reineke, K. Walzer, and K. Leo, Reduced efficiency roll-off in high-efficiency hybrid white organic light-emitting diodes, Appl. Phys. Lett. 92, 053311 (2008).

[24] D. Song, S. Zhao, and H. Aziz, Modification of exciton lifetime by the metal cathode in phosphorescent oleds, and implications on device efficiency and efficiency roll-off behavior, Adv. Funct. Mater. 21, 2311 (2011).

[25] C. Diez, T. C. G. Reusch, E. Lang, T. Dobbertin, and W. Brütting, Highly stable charge generation layers using caesium phosphate as n-dopants and inserting interlayers, J. Appl. Phys. 111, 103107 (2012).

[26] T. D. Schmidt, D. S. Setz, M. Flämmich, B. J. Scholz, A. Jaeger, C. Diez, D. Michaelis, N. Danz, and W. Brütting, Degradation induced decrease of the radiative quantum efficiency in organic light-emitting diodes, Appl. Phys. Lett. 101, 103301 (2012).

[27] W. Brütting, J. Frischeisen, T. D. Schmidt, B. J. Scholz, and C. Mayr, Device efficiency of organic light-emitting diodes: Progress by improved light outcoupling, Phys. Status Solidi A 210, 44 (2013). 
[28] B. Lüssem, M. Riede, and K. Leo, Doping of organic semiconductors, Phys. Status Solidi A 210, 9 (2013).

[29] See the Supplemental Material at http://link.aps.org/ supplemental/10.1103/PhysRevApplied.3.024008 for details regarding characterization of the electrical properties of the doped transport layers and the thickness of the space charge region via impedance spectroscopy as well as a detailed quenching analysis for all individual ETL thicknesses.

[30] S. L. M. van Mensfoort, V. Shabro, R. J. de Vries, R. A. J. Janssen, and R. Coehoorn, Hole transport in the organic small molecule material $\alpha$-npd: Evidence for the presence of correlated disorder, J. Appl. Phys. 107, 113710 (2010).

[31] M. Flämmich, J. Frischeisen, D. S. Setz, D. Michaelis, B. C. Krummacher, T. D. Schmidt, W. Brütting, and N. Danz, Oriented phosphorescent emitters boost oled efficiency, Org. Electron. 12, 1663 (2011).

[32] D. S. Setz, T. D. Schmidt, M. Flämmich, S. Nowy, J. Frischeisen, B. C. Krummacher, T. Dobbertin, K. Heuser, D. Michaelis, N. Danz, W. Brütting, and A. Winnacker, Comprehensive efficiency analysis of organic light-emitting devices, J. Photon. Energy 1, 011006 (2011).

[33] S. Reineke, G. Schwartz, K. Walzer, and K. Leo, Reduced efficiency roll-off in phosphorescent organic light emitting diodes by suppression of triplet-triplet annihilation, Appl. Phys. Lett. 91, 123508 (2007).
[34] K. C. Kao and W. Hwang, Electrical Transport in Solids (Pergamon Press, New York, 1981).

[35] M. A. Lampert and P. Mark, Current Injection in Solids (Academic Press, New York, 1970).

[36] S.-W. Liu, J.-H. Lee, C.-C. Lee, C.-T. Chen, and J.-K. Wang, Charge carrier mobility of mixed-layer organic lightemitting diodes, Appl. Phys. Lett. 91, 142106 (2007).

[37] N. C. Giebink and S. R. Forrest, Quantum efficiency rolloff at high brightness in fluorescent and phosphorescent organic light emitting diodes, Phys. Rev. B 77, 235215 (2008).

[38] S. Mladenovski, S. Reineke, and K. Neyts, Measurement and simulation of exciton decay times in organic lightemitting devices with different layer structures, Opt. Lett. 34, 1375 (2009).

[39] W. C. H. Choy and H. H. Fong, Comprehensive investigation of absolute optical properties of organic materials, J. Phys. D 41, 155109 (2008).

[40] R. Meerheim, S. Scholz, S. Olthof, G. Schwartz, S. Reineke, $\mathrm{K}$. Walzer, and K. Leo, Influence of charge balance and exciton distribution on efficiency and lifetime of phosphorescent organic light-emitting devices, J. Appl. Phys. 104, 014510 (2008).

[41] J. Kalinowski, W. Stampor, J. Mężyk, M. Cocchi, D. Virgili, V. Fattori, and P. Di Marco, Quenching effects in organic electrophosphorescence, Phys. Rev. B 66, 235321 (2002). 